\title{
An experimental check on Stevens's explanation of the partition paradox by successive doubling and bisecting
}

\author{
SERGIO CESARE MASIN \\ University of Padua, Padua, Italy
}

\begin{abstract}
Scaling methods based on direct estimation of sensory ratios and intervals give discrepant results (the partition paradox). Stevens's explanation of this discrepancy was tested here. Given a psychological magnitude, $\psi$, observers were required to select a magnitude $2 \psi$, and subsequently to bisect the interval between $\psi$ and $2 \psi$. Stevens's hypothesis predicts that the bisected psychological magnitude falls between $1.41 \psi$ and $1.50 \psi$. The hypothesis is not substantiated, since a bisected magnitude of $1.55 \psi$ was obtained. Furthermore, observers had to bisect the interval between $\psi$ and an imagined magnitude of $2 \psi$. The results show that observers are able to produce reliably both a visual and a representational double.
\end{abstract}

Ratio scaling is based on the assumption that the human observer is capable of directly reporting sensory ratios between two values of a perceptual variable. The method of fractionation or multiplication requires the observer to estimate, or produce, fractions or multiples (ratios) of given values of a perceptual variable. Interval scaling is based on the assumption that the observer is capable of directly reporting sensory intervals. The method of bisection requires the observer to set the value of a perceptual variable until it is midway between two fixed values of the same perceptual variable, thus producing two equal-appearing intervals.

Typically, the exponent of a power function obtained by the method of bisection is smaller than the exponent obtained by a method based on ratio scaling (Stevens, 1955). Thus, methods based on direct estimation of sensory intervals and ratios do not give consistent results (the partition paradox). According to Stevens (Stevens, 1971, 1975; Stevens \& Davis, 1938), this discrepancy originates in the method of bisection. In bisecting a sensory interval, the observer can adopt two different strategies: He can set the middle value midway between the two end values to obtain two equal-appearing intervals, or he can set the middle value so that the ratio of the middle value to the lower value equals the ratio of the higher value to the middle value. The actual performance, Stevens argued, represents a compromise between these two possibilities.

In an experiment by Garner (1954), a group of observers set a variable stimulus so that the corresponding perceptual value, $\psi_{v}$, stood in the middle of the

I wish to thank Martha Teghtsoonian for a careful reading of the first draft of this manuscript. two perceptual values $\psi_{\mathrm{i}}$ and $\psi_{\mathrm{j}}$. The setting was carried out under two conditions. In one condition, the observers were instructed to set the variable stimulus to produce equal distances, that is, $\psi_{\mathrm{v}}-\psi_{\mathrm{i}}=\psi_{\mathrm{j}}-\psi_{\mathrm{v}}$. In the other condition, they had to set the variable stimulus to produce equal ratios, that is, $\psi_{\mathrm{v}} / \psi_{\mathrm{i}}=$ $\psi_{\mathrm{j}} / \psi_{\mathrm{v}}$. For most observers, the selected value for the variable stimulus was the same under the two conditions. If an estimate of the values $\psi_{i}$ and $\psi_{j}$ were at our disposal, we would be in a position to know whether the observer under the bisection task approximated a setting in terms of equal distances or ratios.

Stevens's hypothesis may be tested by requiring the observer to double a given sensory magnitude, $\psi$, and subsequently to bisect the interval between $\psi$ and $2 \psi$. If it is assumed that the observer is capable, at least approximately, of evaluating the sensory ratio 2 , and if he attempts to make two equalappearing intervals, the bisecting sensory magnitude, $\psi_{b}$, should correspond to the arithmetic mean of the higher, $2 \psi$, and lower, $\psi$, sensory magnitudes, that is $\psi_{\mathrm{b}}=(2 \psi+\psi) / 2=1.5 \psi$. On the other hand, if the observer attempts to equate the ratio of the bisecting magnitude to the lower magnitude with the ratio of the higher magnitude to the bisecting magnitude, then $\psi_{\mathrm{b}} / \psi=2 \psi / \psi_{\mathrm{b}}$, hence $\psi_{\mathrm{b}}=\sqrt{2 \psi \cdot \psi}=1.41 \psi$. In this case, the bisecting sensory magnitude is the geometric mean of the lower and higher sensory magnitudes. And, if the observer makes some compromise, as Stevens hypothesized, then the empirical bisection magnitude should fall between the arithmetic and geometric means.

This test is based on the assumption that the observer is capable of evaluating the sensory ratio 2 . It seemed advisable to check for the robustness of the test by performing the experiment also in a non- 
sensory domain. We may ask whether the same bisection point is produced when, instead of bisecting the interval between two perceptual end values, $\psi$ and $2 \psi$, the observer bisects the interval between a perceptual magnitude, $\psi$, and an imagined magnitude, $2 \psi$.

\section{METHOD}

\section{Observers}

There were 10 observers, 8 psychologists and 2 technicians.

\section{Stimuli}

Using an IBM $370-158$ computer, $22.5 \times 31 \mathrm{~cm}$ rectangles containing dark gray Os were generated on $28 \times 40 \mathrm{~cm}$ white sheets of paper. The rectangles were contoured by pluses ("+"). A rectangle should be understood as a $52 \times 60$ matrix in which a maximum of 3,120 Os could be contained. Each cell had the same probability of an O's appearing in it. All stimuli were shown against a black background $1.5 \mathrm{~m}$ distant and frontal-parallel to the observer. The stimuli were illuminated from above by a $60-\mathrm{W}$ lamp about $70 \mathrm{~cm}$ distant.

\section{Procedure}

There were four sessions. In the first session, the multiplication method (doubling) was used. The observer was shown 10 standard rectangles in random order. The number of $O$ s in a rectangle was $30,48,76,117,159,213,305,437,599$, or 807 . The method of adjustment was used. The variable stimuli were rectangles on white sheets of paper the same in size as the standard stimuli. The number of Os in the stimuli varied from 30 to 1,980 in steps of about 10. The sheets constituting the variable stimuli were contained in a book. The variable stimuli on the right and the standard stimulus on the left were adjacent. The experimenter adjusted the numerosity of the variable stimuli by turning the sheets of the book. The observer's task was to stop the experimenter when the number of Os in the variable stimulus was twice as great as the number of $O$ s in the standard stimulus (point of subjective double). The observer gave two estimates of the double, one with the variable stimuli varying in ascending order and one in descending order.

The second session took place after a 10-15-min rest period, during which the experimenter plotted the points of subjective double on a diagram and graphically fitted a curve through the data points. The standard stimuli containing $30,48,117,213$, 437 , or 807 Os were placed on the observer's left. Each standard was paired with another adjacent standard in which the numerosity of Os was the point of subjective double estimated graphically. On the right of the pair of standard stimuli, there were the variable stimuli contained in a book. The observer's task was to stop the experimenter when the numerousness of $\mathrm{Os}$ in the variable stimulus was midway between the numerousness of Os in the two standards. In other words, the observer had to bisect the interval between a visual numerousness and its visual double (visual bisection point). Two estimates per standard stimulus were given, one obtained by adjusting the variable in ascending order and one in descending order.

In the third session, only the standards containing $30,48,117$, 213,437 , or 807 Os were shown on the observer's left. His task was to stop the experimenter turning over the variable stimuli when the numerousness of $O$ s in the variable stimulus was midway between the visual numerousness of Os in the standard and its imagined double (representational bisection point). Two estimates per standard stimulus were given; these were obtained by adjusting the variable in ascending order, in one case, and in descending order, in the other.

The order of the second and third sessions was reversed for five observers. Subsequent analysis showed that this change in order does not affect the results.

In the fourth session, the observer was again shown all 10 standards. His task was to stop the experimenter when the numerousness of Os in the variable stimulus was the same as that in the standard (point of subjective equality). Two estimates were given, one with the variable stimuli varying in ascending order and one with them varying in descending order.

The total time required to perform the experiment with one observer was about $1.5 \mathrm{~h}$. Before starting the experiment, the observers were asked not to count or compute in any way.

\section{RESULTS}

The results are reported in Table 1. Columns 1 to 5 report, respectively, the actual number of elements in the standard stimulus, $\phi_{\mathrm{N}}$, and the mean values for the point of subjective equality, PSE, the point of subjective double, PSD, the visual bisection point,

Table 1

Matching, Bisecting, and Doubling Responses (Means) and Derived Measures of Subjective Numerousness

\begin{tabular}{|c|c|c|c|c|c|c|c|c|c|c|}
\hline 1 & 2 & 3 & 4 & 5 & 6 & 7 & 8 & 9 & 10 & 11 \\
\hline$\phi_{N}$ & PSE & PSD & VBP & RBP & $\psi_{\text {PSE }}$ & $\psi_{\text {PSD }}$ & $\psi_{\mathrm{VBP}}$ & $\psi_{\text {RBP }}$ & $\frac{\psi_{\mathrm{PSE}}+\psi_{\mathrm{PSD}}}{2}$ & $\sqrt{\psi_{\mathrm{PSE}} \psi_{\mathrm{PSD}}}$ \\
\hline 30 & 35.4 & 71.8 & 57.0 & 54.0 & 1.08 & 1.72 & 1.46 & 1.40 & 1.40 & 1.36 \\
\hline 48 & 52.6 & 111.3 & 94.4 & 87.0 & 1.38 & 2.47 & 2.15 & 2.01 & 1.93 & 1.85 \\
\hline 76 & 77.7 & 183.1 & & & 1.83 & 3.83 & & & & \\
\hline 117 & 124.6 & 266.7 & 212.3 & 225.1 & 2.73 & 5.59 & 4.47 & 4.73 & 4.16 & 3.91 \\
\hline 159 & 167.0 & 369.1 & & & 3.56 & 7.72 & & & & \\
\hline 213 & 210.0 & 454.2 & 337.2 & 345.4 & 4.43 & 9.53 & 7.05 & 7.22 & 6.98 & 6.50 \\
\hline 305 & 305.1 & 668.2 & & & 6.38 & 14.18 & & & & \\
\hline 437 & 431.9 & 816.8 & 626.2 & 642.5 & 9.06 & 17.47 & 13.26 & 13.61 & 13.27 & 12.58 \\
\hline 599 & 593.7 & 993.5 & & & 12.55 & 21.43 & & & & \\
\hline 807 & 813.5 & 1253.3 & 979.6 & 1050.9 & 17.39 & * & 21.11 & 22.72 & & \\
\hline
\end{tabular}

Note-Column 1 reports the number of elements in the target standard stimulus. Columns 2 to 5 report respectively, the point of subjective equality (PSE), the point of subjective double (PSD), the visual bisection point (VBP), and the representational bisection point $(R B P)$. Columns 6 to 9 report the subjective values obtained from the physical values in Columns 2 to 5 . Columns 10 and 11 report the arithmetic and geometric means of the subjective values corresponding to PSE and PSD. Columns 8 or 9 should be compared with Columns 10 and 11. "The value 1253.3, Column 3 and Row 10, exceeds the ambit of assumed validity of the psychophysical function based on the ratio 2 , and therefore is not processed. 
VBP, and the representational bisection point, RBP.

An analysis of variance, in which the factors were the kind of bisection versus the numerosity of elements in the target standard stimulus, shows that the representational and visual bisection points are not different $[F(1,9)=1.55, p>.05]$ and that the interaction of factors is not significant $[F(5,45)=2.05$, $p>.05]$. This shows that the human observer behaves as if he were able to produce an internal representation of the double.

Six points of the psychophysical function relating numerousness, $\psi_{\mathrm{N}}$, to actual number of elements, $\phi_{\mathrm{N}}$, were derived from the function relating the point of subjective double to $\phi_{\mathrm{N}}$ (a parabolic arc of the equation PSD $=14.85+2.82 \phi_{N}-.001 \phi_{N}^{2}, r^{2}=.996$ ) by putting $\psi_{\mathrm{N}}=1$ for $\phi_{\mathrm{N}}=30$. The obtained points were $(30,1),(82,2),(197,4),(426,8),(814,16)$, and $(1237,32)$; these were fitted satisfactorily by the power function $\psi_{\mathrm{N}}=1+.014\left(\phi_{\mathrm{N}}-30\right)^{1.06}, \mathrm{r}^{2}=.991$.

The PSEs, PSDs, VBPs, and RBPs in columns 2 to 5 were transformed, through the psychophysical function, into the corresponding subjective values $\psi_{\mathrm{PSE}}, \psi_{\mathrm{PSD}}, \psi_{\mathrm{VBP}}$, and $\psi_{\mathrm{RBP}}$, which are reported in columns 6 to 9 .

Columns 10 and 11 report the arithmetic and geometric means of $\psi_{\mathrm{PSE}}$ and $\psi_{\mathrm{PSD}}$. A one-way analysis of variance shows that the mean percent deviations of the visual bisecting magnitude from the arithmetic mean, $100\left(\psi_{\mathrm{VBP}}-\psi_{\mathrm{A}}\right) / \psi_{\mathrm{A}}$, where $\psi_{\mathrm{A}}=\left(\psi_{\mathrm{PSE}}+\psi_{\mathrm{PSD}}\right)$ 12 , are statistically invariant with the range of $\phi_{\mathrm{N}} \mathrm{S}$ used $[F(4,36)=1.07, p>.25]$. An overall $z$ test shows that these deviations are statistically different from zero $(z=3.67, p<.001)$.

A one-way analysis of variance also shows that the weight $\delta$ in the bisection model $\psi_{\mathrm{VBP}}=\delta \psi_{\mathrm{PSE}}+$ $(1-\delta) \psi_{P S D}$ is statistically invariant with the size of the intervals used $[F(4,36)=1.33, p>.25]$. Although a more complete design would be necessary to obtain a final answer, this result suggests that the bisection model for numerousness is additive (Anderson, 1970).

\section{CONCLUSION}

A comparison of the magnitudes reported in column 8 or column 9 with those in columns 10 and 11 in Table 1 shows that the bisection magnitude falls outside the range delimited by the arithmetic and geometric means. Therefore, Stevens's hypothesis is not substantiated.

Using the bisection model $\psi_{\mathrm{VBP}}=\delta \psi_{\mathrm{PSE}}+$ $(1-\delta) \psi_{P S D}$, a mean $\delta$ of .42 can be estimated from the data reported in Table 1. Fagot and Stewart (1970) obtained bisection points for a number of intervals on the brightness continuum. The bisection model implies that these bisection points are a function of both $\delta$ and the parameters of the psychophysical function for brightness. Using an iterative least squares procedure, they were able to estimate a mean $\delta$ of .41 or .45 (Fagot \& Stewart, 1970, Table 1), dependent on the assumed form of the psychophysical power function. A $\delta$ less than 1/2 was interpreted as due to a response bias. Fagot and Stewart interpreted their results as due to a perceptual "left dominance" for brightness. This left dominance for brightness has been confirmed independently by Kanizsa and Minguzzi (Note 1). In the present experiment, a similar explanation cannot be invoked to account for the representational bisection. The nature of the response bias still remains to be clarified. Both the multiplication and the bisection methods were used here, whereas in Fagot and Stewart's experiment only the bisection method was used. Since, in both experiments, the obtained $\delta s$ are of the same order of magnitude, it appears plausible that the partition paradox is due to the response bias inherent in the bisection method. Therefore, this suggests that the observer's successive doubling and bisecting operations are mutually consistent.

\section{REFERENCE NOTE}

1. Kanizsa, G., \& Minguzzi, G. F. Contrasto e assimilazione di chiarezza (Report from the Institute of Psychology). Trieste: University of Trieste, 1980.

\section{REFERENCES}

Anderson, N. H. Functional measurement and psychological judgment. Psychological Review, 1970, 77, 153-170.

FAgot, R. F., \& STEWART, M. R. Test of a response bias model of bisection. Perception \& Psychophysics, 1970, 7, 257-262.

Garner, W. R. A technique and a scale for loudness measurement. Journal of the Acoustical Society of America, 1954, 48, 218-224.

Stevens, S. S. The measurement of loudness. Journal of the Acoustical Society of America, 1955, 27, 815-829.

Stevens, S. S. Issues in psychophysical measurement, Psychological Review, 1971, 78, 426-450.

Stevens, S. S. Psychophysics. New York: Wiley, 1975.

Stevens, S. S., \& DAvis, H. Hearing: Its psychology and physiology. New York: Wiley, 1938.

(Manuscript received December 10, 1982; revision accepted for publication May 20, 1983.) 